\title{
OBJECTIVE ANALYSIS OF PRESCHOOLERS PHYSICAL ACTIVITY PATTERNS DURING FREE PLAYTIME
}

\author{
Cory Greever, John Sirard, Sofiya Alhassan, FACSM, University of Massachusetts, \\ Amherst, Amherst, MA
}

\author{
Pediatric Physical Activity Laboratory \\ 30 Eastman Ln. \\ Totman Building Room $131 \mathrm{~B}$ \\ Amherst, MA 01003 \\ (413) 545-6104
}

PURPOSE: Preschool-age children are not meeting the recommended levels of daily physical activity (PA), leading states to implement policies requiring preschools to provide students with 30-60 minutes of free playtime. Preschoolers' activity patterns are intermittent nature. It is possible that current PA policies are not conducive in increasing PA in preschoolers. Currently, there is no data on the temporal patterns of PA intensities during preschoolers' free playtime. The purpose of this study was to examine the temporal patterns of preschoolers' PA levels during a typical 30-minute playtime.

METHODS: Baseline playtime accelerometer counts from three preschool intervention studies were utilized ( $n=363$, age $=4.0 \pm 0.8$ years). Thirty minutes of data were collected using 15-second epochs and classified into sedentary, light and moderate-to-vigorous intensity, and averaged in 5-minute blocks [B1 (0-5minute)-B6 (25-30minute)]. Differences between blocks were analyzed using repeated-measures ANOVA, with a Bonferroni correction for pairwise comparisons. RESULTS: There were significant main effects for percent epochs in sedentary $(F(5,1625)=3.73, p=0.0003)$, light $(F(5$, $1625)=2.65, p=0.02)$ and MVPA (MVPA, $F(5,1625)=2.87, p<0.0001)$. The percent of sedentary epochs significantly decreased from B1 to B4 and never significantly increased. The percent of MVPA epochs significantly increased between B1 and B4, significantly decreasing at B5. CONCLUSIONS: The present data suggest that PA strategies should be implemented to improve the activity level of preschoolers within the first and last 10-minutes of a 30-min playtime or providing more frequent, but shorter playtimes. Additional research is needed to understand the play patterns of preschoolers during shorter/longer playtimes. 